\title{
Optimum Design of Structural Parameters for Thin-walled Metal Container
}

\author{
Liao Hongbo ${ }^{*}$, Yang Dan, Yin Fenglong, Liang Xiaodong, Li Erkang and Sun Ye \\ Northwest Institute of Nuclear Technology, Xi'an 710024, China.
}

\begin{abstract}
In order to further increase the volume, reduce the weight and manufacturing cost, the key structural parameters of thin-walled metal packing container are optimized. The instability conditions under circumferential external pressure and axial load are analyzed, a mathematical model with the constraint of critical instability strength, the maximum volume and minimum mass as the objective is constructed. Multi-objective optimization method with nonlinear constraints is used to solve the key structural parameters, such as wall thickness, diameter and height, and the optimization result is calculated by fgoalattain() function in the Matlab optimization toolbox. The instability pressure test system is constructed, the instability pressure of the optimized thin-wall metal packing container is tested. The results show that the unstable pressure is higher than $120 \mathrm{kPa}$, which are better than the design index.
\end{abstract}

\section{Introduction}

Thin-walled metal packing container is widely used in food packaging industry. It has the advantages of low water transmittance, no light transmission, good gas resistance and so on [1]. The vacuum method can effectively reduce the contact between oxygen and food, thus realizing the long-term preservation of food. The existing thin-walled metal packing containers are mainly manufactured by electroplating thin steel plate (commonly known as tinplate), which has realized mass production and assembly line production [2-4]. In order to further reduce the cost of batch production, it is necessary to strictly control the amount of material used in a single container, so as to ensure that the volume can be maximized and the quality minimized under the condition of resistance to standard atmospheric pressure. This puts forward higher requirements for the structural design of existing containers.

As a kind of pressure vessel, thin-walled metal packing vessel has been studied on the optimization of pressure vessel structure. It mainly focuses on basic structure optimization, structural parameter optimization and mathematical method optimization [5-8]. In the aspect of the basic structure optimization, the thin-walled metal packing container mostly adopts the cylindrical shape, the shape is relatively fixed, so the optimization space is small, and the structural optimization is mainly focused on the optimization of structural parameters and mathematical methods. For the research of structural parameter optimization and mathematical method optimization, the existing literature mainly establishes the mathematical model of structural parameter optimization, which includes objective function, constraint function and design variable. Combining the optimization algorithm of Matlab optimization toolbox or Ansys finite element analysis tool to obtain the value of design variables by solving the established mathematical model of parameter optimization [9-13]. The thin-wall metal packing container needs to be subjected to the pressure load at the standard atmospheric pressure, the precondition of the structure optimization is to ensure that the instability is not generated under a standard atmospheric pressure condition, the minimum instability pressure is required. The mathematical model of the parameters of the design variable is optimized by the thickness, diameter and height, and the solution method is sought to ensure the global convergence of the target parameter value.

The research ideas of this paper are as follows: firstly, the basic structure of thin-walled metal packing container is introduced briefly, and the optimization design problem is put forward; then the mathematical model of optimization design is constructed, and the optimal design parameter value is obtained by using Matlab optimization toolbox; finally, the key performance index of thin-wall metal packaging container is tested to verify whether it meets the design requirements.

\section{Structure introduction}

The thin-wall metal packing container studied in this paper refers to the metal tank made of metal material with thickness between $0.15 \sim 0.30 \mathrm{~mm}$. The main materials used in this container are tinplate sheet, Wuxi

* Corresponding author: 13975890826@163.com 
thin steel plate, aluminum alloy plate, Chromium plated and low carbon steel sheets. The structure is divided into three and two pieces of tanks, the three pieces of tank is composed of a body, a top cover and a bottom cover. The body of this tank is welded by resistance welding, and the edges of the top cover and bottom cover are coated with sealant. The two pieces of tank is composed of a bottom body and a top cover, the sealing connection between the top cover and the body is the same as that between the three pieces of tank [1]. The typical structure of thin-walled metal packing container is shown in Fig. 1.

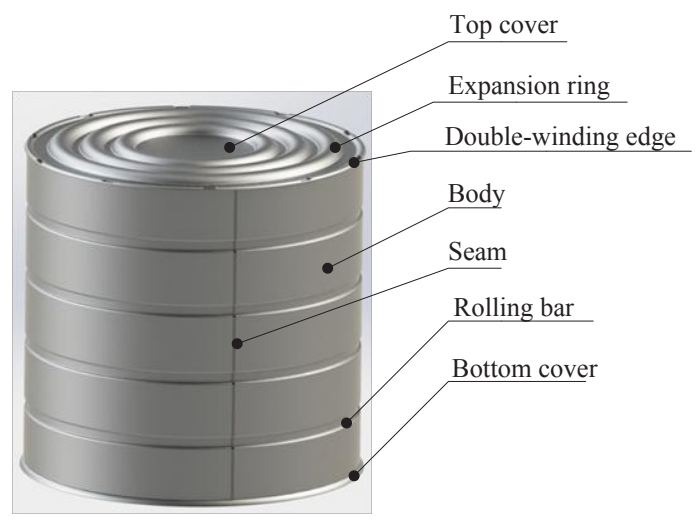

Fig. 1. Typical structure of thin-walled metal packing container.

In the thin-walled metal packing container, under the condition of determining the basic shape of the structure, by selecting reasonable parameters such as diameter, thickness and height, the effective volume of the container is large and the weight is light. At the same time, it can ensure that the structure does not lose its stability under a standard atmospheric pressure load, so as to achieve the purpose of maximum volume, minimum weight or minimum cost.

\section{Structural parameter optimization}

\subsection{Problem description}

The thin-walled metal packing container is made of the same material as the tank body, the top cover and the bottom cover. The design pressure is $P=101 \mathrm{kPa}$, the material is tinplate, and the elastic modulus $E$ is $206 \mathrm{GPa}$, poisson ratio $u$ is 0.3 . According to the characteristics of existing materials and processing technology, the reference range of wall thickness is $b \in[0.15 \sim 0.30]$ $\mathrm{mm}$, diameter reference range is $D \in[80 \sim 160] \mathrm{mm}$, height reference range $H \in[100 \sim 180] \mathrm{mm}$. In order to ensure that the container does not lose stability under external pressure, the minimum critical instability pressure $(P c r) \geqslant c \times P u, c$ is the safety factor. The objective of optimization is that the effective volume of thin-walled metal packing container is the largest, the mass is the minimum, the maximum effective volume is guaranteed firstly, and the optimization parameters are container thickness $b$, diameter $D$ and height $H$.

\subsection{Mathematical model construction}

The optimal design of structural parameters is based on the mathematical model of structural parameter, and the optimal parameter value is obtained by using the optimization algorithm. The basic mathematical model for parameter optimization design can be expressed as

$$
\left\{\begin{array}{l}
\min F(\mathbf{X})=F\left(x_{1}, x_{2} \ldots \ldots x_{n}\right) \\
\text { find } \mathbf{X}=\left[x_{1}, x_{2} \ldots \ldots x_{n}\right]^{\mathrm{T}} \in R \\
\text { s.t. } g_{i}(\mathbf{X})=g\left(x_{1}, x_{2} \ldots \ldots x_{m}\right) \leq 0 \\
i=(1,2, \ldots \ldots . m) \\
h_{j}=h\left(x_{1}, x_{2} \ldots \ldots x_{k}\right)=0 \\
j=(1,2, \ldots \ldots k)
\end{array}\right.
$$

Where $F(\mathbf{X})$ is the objective function, $\mathbf{X}$ is the design variable, $g_{i}(\mathbf{X}), h_{j}(\mathbf{X})$ is the constraint condition, and $n, m$ and $k$ are the number of variables. According to formula (1), the mathematical model of structural parameter optimization of thin-walled metal packing container is established.

\subsubsection{Design variables and objective functions}

The dimension parameters to be optimized include wall thickness $b$, diameter $D$ and height $H$, so the design variables are defined as

$$
\mathbf{X}=\left[x_{1}, x_{2}, x_{3}\right]^{\mathrm{T}}=[b, D, H]^{\mathrm{T}}
$$

The objective function is defined as the maximum effective volume and the minimum mass respectively, which are expressed as

$$
\left\{\begin{array}{l}
\max F_{1}(\mathbf{X})=\pi(D / 2)^{2} H \\
\min F_{2}(\mathbf{X})=\rho \pi(D+b) H t+2 \rho \pi(D / 2+b)^{2} b
\end{array}\right.
$$

where $\rho$ is the density of material.

\subsubsection{Constraint condition}

The size constraint is expressed as

$$
\left\{\begin{array}{l}
80 \leq D \leq 160 \\
0.15 \leq b \leq 0.3 \\
100 \leq H \leq 180
\end{array}\right.
$$

When the load increases to a certain value, the container will suddenly lose its original shape, being flattened or corrugated. After the load is unloaded, the container cannot be restored to its original state. For a thin-walled container with a small ratio of wall thickness to diameter, the compressive stress of thin wall is usually lower than the proportional limit of the material, which 
is called elastic instability [14]. For the elastic instability under various load conditions, the failure stress of a single load is generally determined firstly, the ratio of the stress caused by a single load to the corresponding failure stress is calculated, and then the sum of all the ratios is obtained. If the ratio is less than 1 , there will be no instability.

The elastic stability condition of thin-walled metal packing container under multiple loads is expressed as

$$
\frac{\delta}{\delta_{c r}}+\frac{P}{P_{c r}}<1
$$

Where $P_{c r}$ is the critical unstable pressure, $\delta_{c r}$ is the critical stress under the action of circumferential external pressure and axial load. $\delta$ is the stress produced by axial force, which can be decomposed into stress $\delta_{p}$ from uniform pressure $P$ and stress $\delta_{F}$ from concentration pressure $F$. The formulas of $P_{c r}, \delta_{c r}$ and $\delta$ are given respectively.

$P_{c r}$ is related to the height of thin-walled container, it is a characteristic length $H$ as the boundary between long thin-walled container and short thin-walled container. When $H>H c r$, it belongs to long-thin-walled container, and when $H<H c r$, it belongs to short thin-walled container. The formula of $\mathrm{Hcr}$ is

$$
H_{c r}=1.17 D \sqrt{\frac{D}{b}}
$$

According to the size limit data of thin-walled container and the above calculation method, it can be determined that the thin-walled container designed in this paper is a short thin-walled container, and the formula for calculating the critical unstable pressure of the short thin-walled container is expressed as

$$
P_{c r}=\frac{2.59 E b^{2}}{H D \sqrt{D / b}}
$$

$\delta_{c r}$ is the critical stress under the action of circumferential external pressure and axial load [14]. According to the theoretical and experimental results of nonlinear large deflection, the formula is

$$
\delta_{c r}=0.5 \frac{E b}{D}
$$

$\delta_{p}$ is the stress produced by uniform pressure $P$, and the radial stress between the bottom cover and the body of thin-walled vessel is much larger than the film stress, so the stress is calculated by the combination of circumferential stress and radial stress. The formula is

$$
\delta_{p}=\sqrt{\left(\sum \delta_{x}\right)^{2}+\left(\sum \delta_{\theta}\right)^{2}}=\frac{1.047 P D}{b}
$$

$\delta_{F}$ is the stress produced by axial concentrated pressure $F$, the formula is

$$
\delta_{F}=\frac{P D}{4 b}
$$

$\delta$ is obtained by summing (9) and (10), the formula is

$$
\delta=\frac{P D}{4 b}+\frac{1.047 P D}{b}=\frac{1.3 P D}{b}
$$

By replacing the formulas (7), (8) and (11) with the formula (5), the following formulas are obtained

$$
\frac{2.6 P D^{2}}{E b^{2}}+\frac{0.39 P H D \sqrt{D}}{E b^{2} \sqrt{b}}<1
$$

Based on the above analysis, the mathematical model of structural parameter optimization for thin-walled metal packing container is obtained.

$$
\left\{\begin{array}{l}
\mathbf{X}=\left[x_{1}, x_{2}, x_{3}\right]^{\mathrm{T}}=[b, D, H]^{\mathrm{T}} \\
\min F_{1}(\mathbf{X})=-\pi\left(x_{2} / 2\right)^{2} x_{3} \\
\min F_{2}(\mathbf{X})=\rho \pi\left(x_{2}+x_{1}\right) x_{1} x_{3}+2 \rho \pi\left(x_{2} / 2+x_{1}\right)^{2} x_{1} \\
g(\mathbf{X})=\frac{2.6 P x_{2}^{2}}{E x_{1}^{2}}+\frac{0.39 P x_{3} x_{2} \sqrt{x_{2}}}{E x_{1}^{2} \sqrt{x_{1}}}-1<0 \\
0.15 \leq x_{1} \leq 0.3 \\
80 \leq x_{2} \leq 160 \\
100 \leq x_{3} \leq 180
\end{array}\right.
$$

\subsection{Process analysis of matlab optimal design}

The formula (13) is a multi-objective optimization problem with nonlinear constraints and can be solved by using the fgoalattain() function in the Matlab optimization toolbox. The fgoalattain () function is based on the goal attainment method algorithm, and the optimization principle is to minimize the difference between the objective and the function. The core of the algorithm is to change the multi-objective optimization problem into a single-objective optimization problem by setting the goal-objective judgement vector and weightvector [15]. It is very important to set the initial value of the design vector $\mathbf{X}_{\mathbf{0}}$, which is related to whether the result of the optimization solution converges to the global solution. The flow chart of structural parameter optimization design of thin-walled metal packing container using Matlab optimization toolbox is shown in Fig. 2.

According to the above mentioned process, the program was programmed in Matlab, and the result of the 10th calculation was selected as the final result of the operation. The result was shown that the mass of container is $153 \mathrm{~g}$, the wall thickness of container is $0.27 \mathrm{~mm}$, the diameter of container is $135.5 \mathrm{~mm}$, and the height of container is $102.8 \mathrm{~mm}$. In order to further verify the optimization design results, the thin-wall metal 
packing container samples shall be subjected to the instability pressure test.

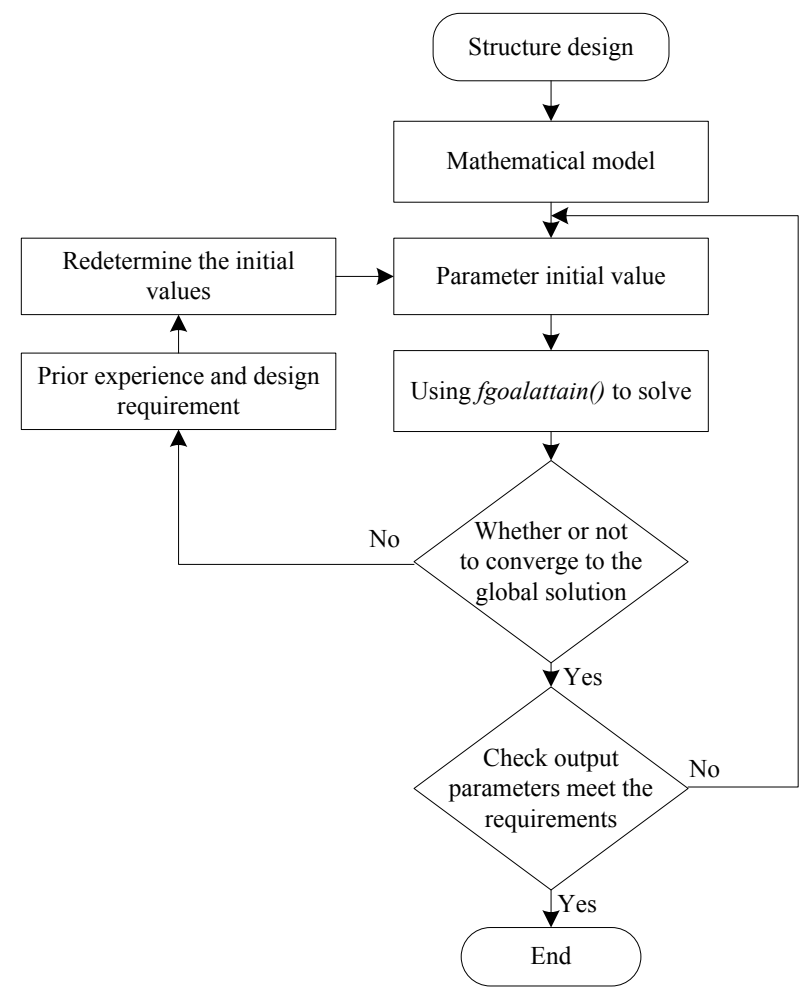

Fig. 2. Flow chart of structural parameters optimal design.

\section{Unstable pressure test}

\subsection{Test system composition}

The unsteady pressure determines the compressive strength of the thin-walled metal packing container. The testing principle is that the container is placed in the test chamber and the gas mass flow controller is used to increase the pressure slowly. The schematic diagram of the structure of the test system is shown in Fig. 3.

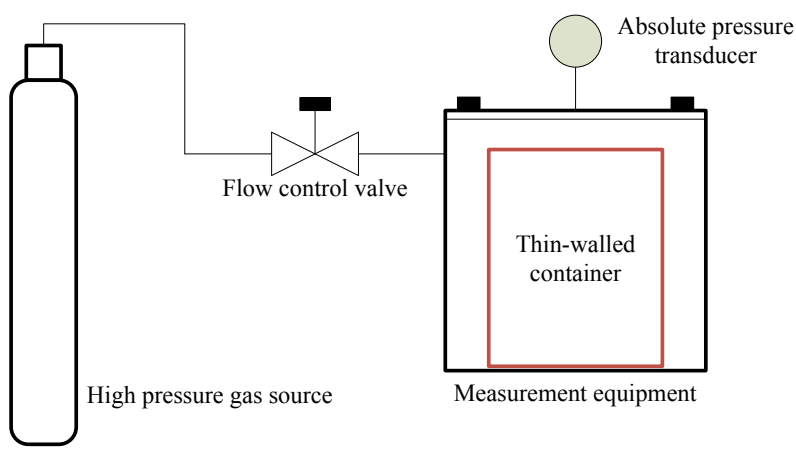

Fig. 3. Schematic diagram of unstable pressure test system.

\subsection{Test result analysis}

When the container is unstable, the volume of the container decreases dramatically and the pressure curve of the interlayer will decrease. Then the difference between the interlayer pressure and the pressure before the instability $(4 \mathrm{kPa})$ is the value of the unstable pressure. The result is shown in Fig. 4.

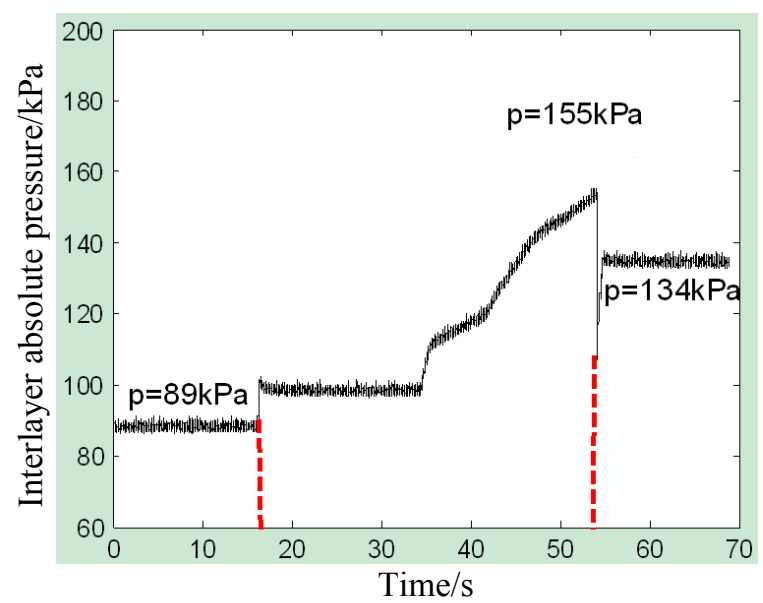

Fig. 4. Absolute pressure curve of cavity interlayer.

From Fig. 4, it can be seen that the absolute pressure of the interlayer of the test cavity is increasing continuously. When it reaches $155 \mathrm{kPa}$, the container loses stability, which volume decreases significantly, and the absolute pressure of the interlayer decreases suddenly. Seven sample containers were selected for repeated tests. The results are shown in Fig. 5. It can be seen that the unstable pressure of thin-walled metal packing container is between $132 \mathrm{kPa} \sim 165 \mathrm{kPa}$, which is higher than the design index $120 \mathrm{kPa}$. Because the body of thin-walled metal packing container is strengthened, the expansion ring is increased on the top cover and bottom cove, and the overall stiffness of the vessel is increased, so the unstable pressure is higher than the design value.

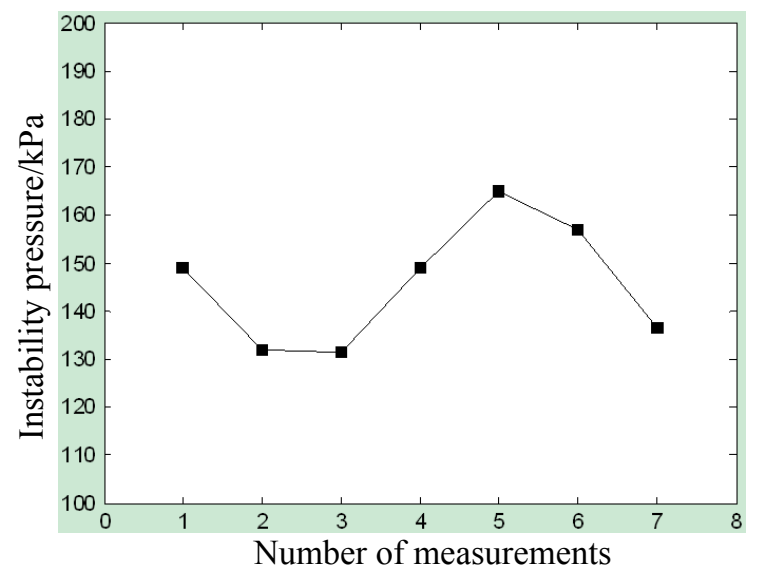

Fig. 5. Repeatability test data of unstable pressure.

It can also be seen from Fig. 4 that the absolute pressure of the interlayer after the container is unstable remains stable, indicating that the container is deformed without obvious damage, and the morphology of the container before and after the instability is compared as shown in Fig. 6. 


\section{References}

\section{Conclusion}

In this paper, the optimization design of structural parameters and the performance test of thin-walled metal packing container are studied. The mathematical model of structural parameter optimization of thin-walled metal packing container is constructed and solved by Goal Attainment method. The test results show that the instability pressure is higher than $120 \mathrm{kPa}$, which meets the design requirements.

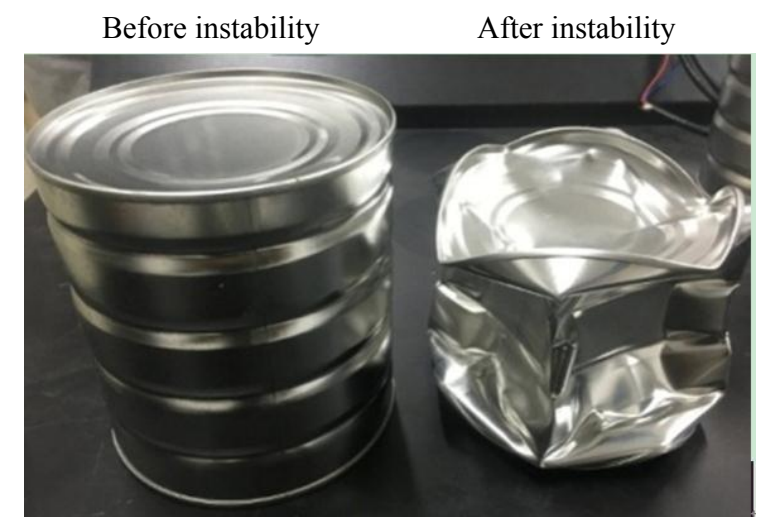

Fig. 6. Morphological comparison before and after instability.
1. Y. Mei, R.Q. Li, Mech. Mer. \& Dev. 2, 3 (2005)

2. W.Y. Guo, SHP. 4, 3 (2016)

3. J.C. Liu, Contemp. Chem. Ind. 6, 5 (2006)

4. R. Odette, K. Nanstad, J. Miner. Met. Mat. Soci. 11, 9 (2011)

5. R. Datta, D. Mukerje, S. Mishrae, Mat. Eng. \& Perfor. 7, 3 (1998)

6. Ch. Chen, H. Li, Y. L. Lei, G. Chem. Ind. 45, 3 (2017)

7. D. Gao, ZH. SH. Cheng, T. L. Wei, Trans. CN. Soci. Agricul. Mach. 3, 3 (2000)

8. F. E. Baginski, J. Num. Analy. 3, 6 (1994)

9. S. M. Carr, W. E. Lawrence, M. N. Wybourne, IEEE Trans. Nanotech. 6, 5 (2005)

10. J. Y. Tan, SH. Liu, J. Hu. Ins. Sci, Tech. 3, 5 (2016)

11. B. M. Ding, ASME VIII-1 \& VIII-2, (1995)

12. B. Liu, R. T. Haftka, M. A. Akgun, Struct. \& Multidiscip. Optim. 2, 10 (2000)

13. S. Guo, W. Chen, D. Cui, AIAA J. 12, 6 (2006)

14. J. Y. Zheng, Chemical Industry Press, (2001)

15. N. Ravikumar, C. Shekarbesta, M. Chidambaram, Trend. Ind. Mea. \& Auto. 5 (2017) 